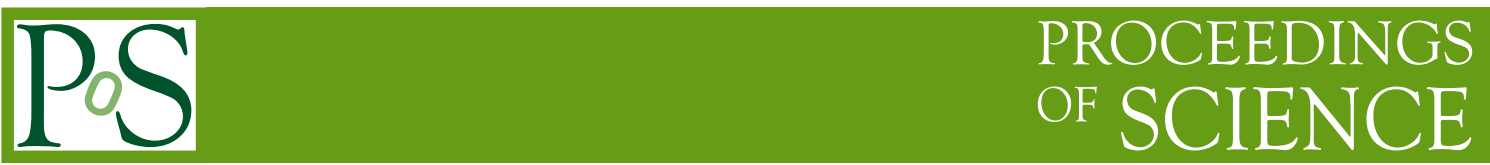

\title{
COMPASS polarized Drell-Yan experiment
}

\author{
N. DOSHITA* \\ (for the COMPASS collaboration) \\ Yamagata University, Japan \\ E-mail: norihiro.doshita@cern.ch
}

The COMPASS II started at 2012 that includes polarized Drell-Yan program with a polarized solid target. The availability of pion beam provides an access to the Drell-Yan physics throughout the process where quark(target)-antiquark(beam) pair annihilates electromagnetically with a production of dilepton pair. Study of angular dependencies of the Drell-Yan process cross-section allows us to access to parton distribution functions (PDFs) or, more precisely, a convolutions of various PDFs. The transversely polarized target together with negative pion beam is an important feature of the COMPASS Drell-Yan experiment, that provides us with unique data on transverse momentum dependent (TMD) PDFs.

After a plot run in 2014, the experiment has just started in 2015. The role of the Drell-Yan experiment at COMPASS in TMD PDFs study, with a comparison to semi-inclusive deep inelastic scattering experiment, is described. The experimental set-up, the status of the data taking in 2015 and preliminary analysis results in the 2014 run are presented.

XVIth International Workshop in Polarized Sources, Targets, and Polarimetry, PSTP2015,

14-18 September 2015,

Bochum, Germany

${ }^{*}$ Speaker. 


\section{Introduction}

The COMPASS experiment at CERN has been taking data since 2002 using either muon or hadron beams with a longitudinally or transversely polarized solid target, liquid hydrogen or heavy nuclear targets. And many interesting and impressive results on nucleon spin structure and hadron spectroscopy have been published. The COMPASS Collaboration has recently started the second phase for additional measurements in the next years from 2012 as COMPASS II [1]. It includes studies of polarized Drell-Yan program, Generalized Parton Distribution (GPD) program and pion and kaon polarizabilities program.

\section{The experimental set up}

CERN SPS M2 beam line delivers hadron or naturally polarized positive and negative muon beams in the energy range between 50 and $280 \mathrm{GeV}$. The muon beam polarization is about $80 \%$ at $160 \mathrm{GeV}$. The COMPASS experiment uses those beams with a longitudinally or transversely polarized solid target, liquid hydrogen or heavy nuclear targets [2, 3, 4].

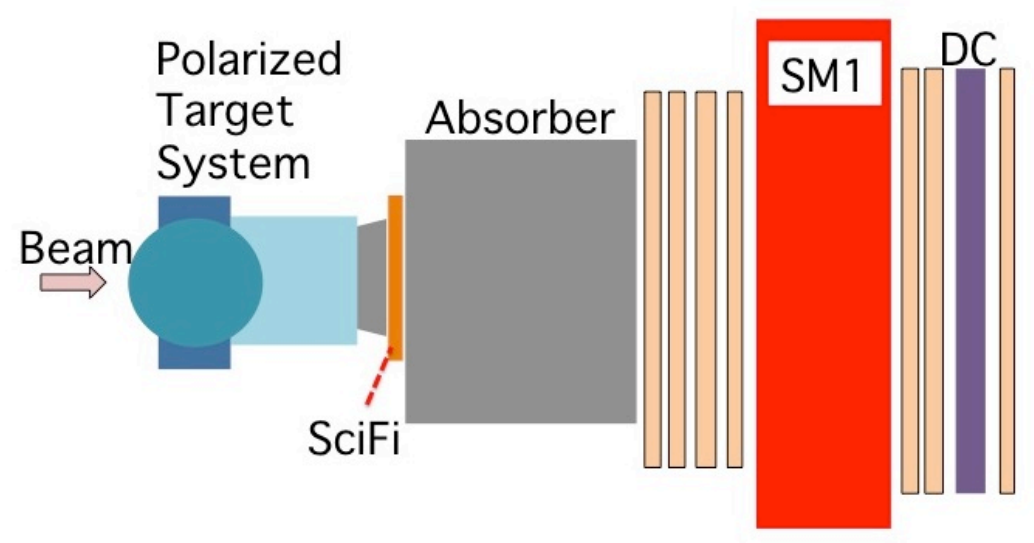

Figure 1: A schematic top view of COMPASS apparatus between the target and the new DC

The Drell-Yan measurement requires a negative pion beam and a transversely polarized proton target. We use the negative pion beam of $190 \mathrm{GeV}$ mixed with negative kaon and positron $\left(\pi^{-} 97\right.$ $\%, \mathrm{~K}^{-} 2.5 \%$ and $\bar{P} 0.5 \%$ ). Those beam particles are identified by CEDAR (CErenkov Differential counter with Achromatic Ring focus). The CEDAR is placed in the downstream of the M2 line. The COMPASS polarized target system needed to be modified for this purpose. We use two cells of $55 \mathrm{~cm}$ long with a $4 \mathrm{~cm}$ diameter and the distance between the cells is $20 \mathrm{~cm}$ because of vertex resolution. The target cells are filled by ammonia beads of a few millimeters size as proton target material. The detail polarized target system is described in [5]. The target system moved $2.3 \mathrm{~m}$ upstream to install a hadron absorber behind the polarized target (Fig 1). At the beam intensity of $1 \times 10^{8} / \mathrm{s}$ this absorber is mandatory, in order to keep the occupancies in the first detector planes at an acceptable level, and to reduce the combinatorial background from pion decays into muons. The choice of material, length and geometry of the absorber must be such that it minimizes the muon multiple scattering, while maximizing the hadron stopping power, and keeping the radiation 
levels within the allowed limits. A beam plug inside the absorber is also mandatory, in order to stop the beam that did not interact in the target. The configuration for the hadron absorber is a length along the beam direction of $236 \mathrm{~cm}$ in total. The absorber is made of alumina $\left(\mathrm{Al}_{2} \mathrm{O}_{3}\right)$ with a layer of stainless steel frames. Two beryllium and one polyethylene sheets are also installed in the most downstream part to stop produced neutrons. The tungsten beam plug with a $140 \mathrm{~cm}$ long and a $9.5 \mathrm{~cm}$ diameter is inserted inside the absorber. A $36 \mathrm{~cm}$ long conical alumina nose of the absorber enters into the target magnet downstream window. A Scintillating fiber station (SciFi) with 3 planes is newly installed in the gap between the conical absorber and the main one in order to improve the vertex resolution. The station is constructed by $1 \mathrm{~mm}$ diameter fibers that has 700 ps time resolution. A new drift chamber (DC) covering larger area is also installed behind the first spectrometer magnet (SM1) for tracking improvement in 2015.

\section{Polarized Drell-Yann program}

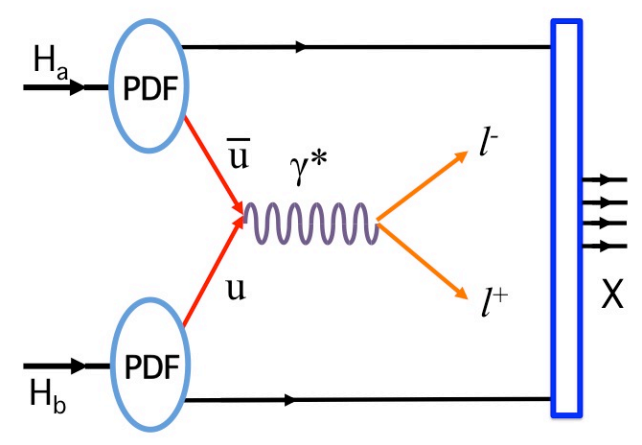

Figure 2: Feynman diagram of the Drell-Yan process

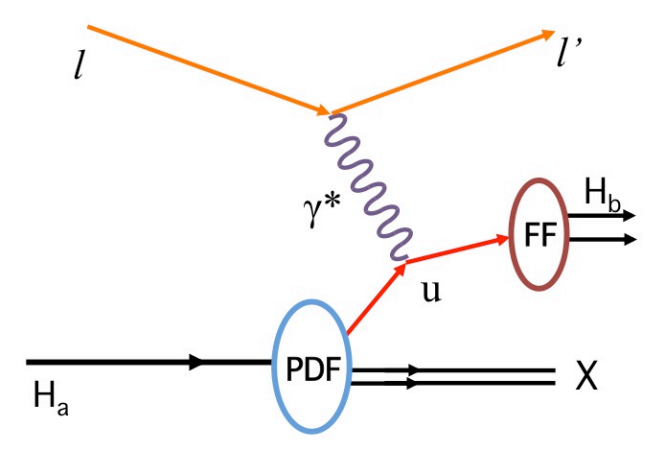

Figure 3: Feynman diagram of the SIDIS process

The availability of pion beam provides an access to the Drell-Yan physics, i.e. to the process where quark(target)-antiquark(beam) pair annihilates electromagnetically with a production of dilepton pair (Fig. 2). Study of angular dependencies of the Drell-Yan process cross-section allows us to access to parton distribution functions (PDFs) or, more precisely, a convolutions of various PDFs. The possibility to use a transversely polarized target together with negative pion beam is an important feature of the Drell-Yan program. This program provides us with unique data on transverse momentum dependent (TMD) PDFs.

The leading order expansion of the single polarized Drell-Yan cross section is

$$
\begin{aligned}
\frac{d \sigma}{d^{4} q d \Omega}= & \frac{\alpha^{2}}{F q^{2}} \hat{\sigma}_{U}\left\{\left(1+D_{\left[\sin ^{2} \theta\right]} \underline{\underline{A_{U U}^{\cos 2 \phi}}} \cos 2 \phi\right)\right. \\
& +\left|\vec{S}_{T}\right|\left[\underline{\underline{A_{U T}^{\sin \phi_{s}} \sin \phi_{s}}}\right. \\
& +D_{\left[\sin ^{2} \theta\right]}\left(\underline{\underline{A_{U T}^{\sin }\left(2 \phi+\phi_{s}\right)}} \sin \left(2 \phi+\phi_{s}\right)\right. \\
& +\underline{\left.\left.\left.\underline{A_{U T}^{\sin \left(2 \phi-\phi_{s}\right)}} \sin \left(2 \phi-\phi_{s}\right)\right)\right]\right\}}
\end{aligned}
$$


where $D$ is depolarization factor, $S$ is target spin component, $\hat{\sigma}_{U}$ is a part of the cross-section surviving integration over $\phi$ and $\phi_{s}$ and $F=4 \sqrt{\left(P_{a} \cdot P_{b}\right)^{2}-M_{a}^{2} M_{b}^{2}}$. The azimuthal asymmetries $A_{U, L, T}^{f\left(\phi, \phi_{s}\right)}$ are described with a convolution of two PDFs as

$$
\begin{aligned}
A_{U U}^{\cos 2 \phi} & :(\mathbf{B M})_{\pi} \otimes(\mathbf{B M})_{p} \\
A_{U T}^{\sin \phi_{s}} & :\left(f_{1}\right)_{\pi} \otimes(\text { Sivers })_{p} \\
A_{U T}^{\sin \left(2 \phi+\phi_{s}\right)} & :(\mathbf{B M})_{\pi} \otimes(\text { Pretzelosity })_{p} \\
A_{U T}^{\sin \left(2 \phi-\phi_{s}\right)} & :(\mathbf{B M})_{\pi} \otimes(\text { Transversity })_{p},
\end{aligned}
$$

where $\mathbf{B M}$ means Boer-Mulders functions.

One of the main motivations of the program is to study the universality of TMD PDFs. Because Sivers $\left(f_{1 T}^{\perp}\right)$ and Boer-Mulders $\left(h_{1}^{\perp}\right)$ PDFs are "Time-reversal odd", they are expected to change the sign when measured from SIDIS or from DY as

$$
\left.f_{1 T}^{\perp}\right|_{D Y}=-\left.\left.f_{1 T}^{\perp}\right|_{S I D I S} \quad h_{1}^{\perp}\right|_{D Y}=-\left.h_{1}^{\perp}\right|_{S I D I S}
$$

The Sivers asymmetry in SIDIS was measured in COMPASS in 2007 and 2010 [6, 7]. We have the opportunity to test this sign change using the same spectrometer and the transversely polarized target at COMPASS. The azimuthal asymmetries in SIDIS are described with a convolution of a PDF and a Fragmentation Function (FF) (Fig. 3).

A luminosity of $L=2.3 \times 10^{32} \mathrm{~cm}^{-2} \mathrm{~s}^{-1}$ can be obtained with a beam intensity of $I_{\text {beam }}=$ $1 \times 10^{8} / \mathrm{s}, 190 \mathrm{GeV} / \mathrm{c} \pi^{-}$beam, and with a $110 \mathrm{~cm}$ long polarized proton target. Assuming 1 years (140 days) of data taking, one can obtain the statistical accuracy of 0.013 in the asymmetry of $A_{U T}^{s i n \phi_{s}}$ at the dimuon mass range of $4<M_{\mu \mu}<9 \mathrm{GeV} / \mathrm{c}^{2}$ where is negligible small of background contamination (Table 1).

\section{Data taking in 2014 and 2015}

In 2014 we performed a pilot run for 10 days stable data taking with $190 \mathrm{GeV} / \mathrm{c} \pi^{-}$beam on a unploarized target of $\mathrm{NH}_{3}$. The beam intensity was $7 \times 10^{7} / \mathrm{s}$.

The hadron absorber and the beam plug were already installed. Figure 4 shows the vertex distribution along the beam with the dimuons mass of $M_{\mu \mu}>4 \mathrm{GeV} / \mathrm{c}^{2}$. The peaks from the left to the right in the figure represent the two ammonia target material cells, the vertex detector, the aluminum target and the tungsten beam plug. The run was to study combinatorial background and event yields of $\mathrm{J} / \psi$ and Drell-Yan. The combinatorial background and $\mathrm{J} / \psi$ are main background sources (Fig. 5). The data analysis is in process.

We started physics data taking at the end of June in 2015 and continue until the middle of November. The typical beam intensity is about $8 \times 10^{7}$ pions $/ \mathrm{s}$ in about 10 seconds for the duration of 50 seconds SPS cycle. The target polarization direction changes by microwave in Dynamic Nuclear Polarization (DNP) every 2 weeks. It takes about 1 day. 
Table 1: Expected statistical accuracy in the 2015 run

\begin{tabular}{lr}
\hline Asymmetry & Statistical accuracy $\left(4<M_{\mu \mu}<9 \mathrm{GeV} / \mathrm{c}^{2}\right)$ \\
\hline \hline$A_{U U}^{\cos 2 \phi}$ & 0.005 \\
$A_{U T}^{\sin \phi_{s}}$ & 0.013 \\
$A_{U T}^{\sin \left(2 \phi+\phi_{s}\right)}$ & 0.027 \\
$A_{U T}^{\sin \left(2 \phi-\phi_{s}\right)}$ & 0.027 \\
\hline
\end{tabular}

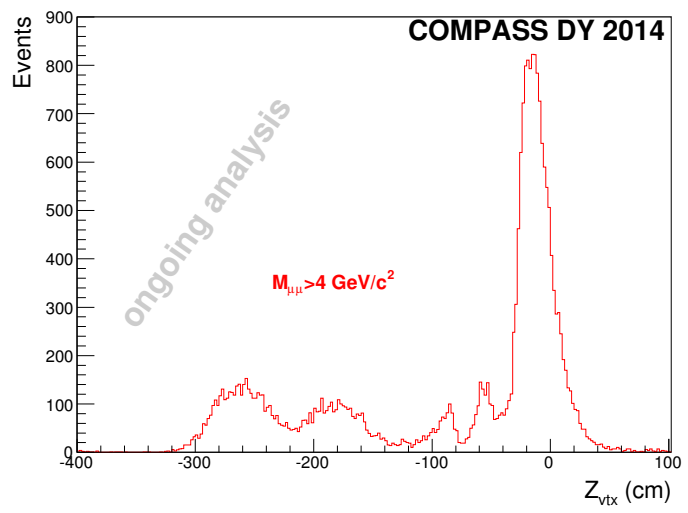

Figure 4: $\mathrm{Z}$ vertex distribution selecting higher mass Figure 5: Dimuon mass distribution and background dimuons of $M_{\mu \mu}>4 \mathrm{GeV} / \mathrm{c}^{2}$.

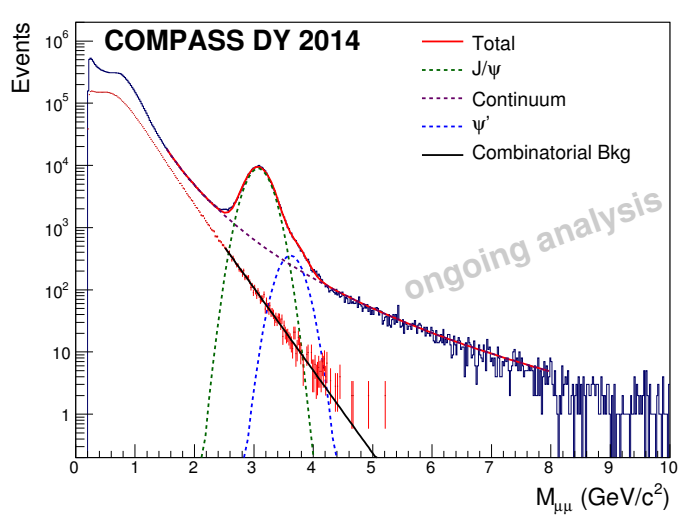

distributions

\section{References}

[1] The COMPASS Collaboration, F. Gautheron et al., COMPASS-II Proposal, CERN-SPSC-2010-014, SPSC-P-340, May 17, 2010.

[2] The COMPASS Collaboration, P. Abbon et al, "The COMPASS experiment at CERN ", Nucl. Instr. and Meth. A 577, pp. 455-518 (2007).

[3] The COMPASS Collaboration, Ph. Abbon et al, "The COMPASS setup for physics with hadron beams", Nucl. Instr. and Meth. A 779, pp. 69-115 (2015).

[4] E. Bielert et al, "A $2.5 \mathrm{~m}$ long liquid hydrogen target for COMPASS ", Nucl. Instr. and Meth. A 746, pp. 20-25 (2014).

[5] J. Koivuniemi et al., these proceedings.

[6] The COMPASS Collaboration, M. G. Alekseev et al, "Measurement of the Collins and Sivers asymmetries on transversely polarised protons", Phys. Lett. B 692, pp. 240-246 (2010).

[7] The COMPASS Collaboration, C. Adolph et al, "II - Experimental investigation of transverse spin asymmetries in $\mu$ - p SIDIS processes: Sivers asymmetries", Phys. Lett. B 717, pp. 383-389 (2014). 